\title{
Anomalías vasculares más frecuentes en pacientes pediátricos. Parte 1: Tumores vasculares
}

\section{Vascular anomalies in childhood. Part 1: Vascular tumors}

\author{
Edna Morán-Villaseñor, ${ }^{1}$ Blanca Lucina Campos-Cabrera, ${ }^{1}$ María Teresa García-Romero, ${ }^{2}$ Carola Durán-McKinster ${ }^{2}$
}

\section{Resumen}

Las anomalías vasculares son un grupo heterogéneo de alteraciones en los vasos sanguíneos y linfáticos. La International Society for the Study of Vascular Anomalies (ISSVA) las clasifica en: tumores y malformaciones vasculares. En este artículo (primera parte de dos) se revisan los principales tumores vasculares en pacientes pediátricos, su fisiopatogenia, manifestaciones clínicas, diagnóstico y tratamiento. Nos enfocaremos en el hemangioma infantil, hemangiomas congénitos, granuloma piógeno, angioma en penacho y hemangioendotelioma kaposiforme.

El hemangioma infantil es el tumor vascular más frecuente, aparece en las primeras 4 semanas de vida y crece con rapidez, para posteriormente involucionar en la etapa preescolar. Se manifiesta como una neoformación vascular, de coloración eritematoviolácea, de tamaño variable. Los hemangiomas congénitos son más raros, están completamente desarrollados al nacimiento porque su crecimiento es intrauterino. El granuloma piógeno es el tumor vascular más frecuente, después del hemangioma infantil, y se manifiesta como una neoformación de color rojo-violáceo, exofítica; es de crecimiento rápido y sangra fácilmente. El angioma en penacho y el hemangioendotelioma kaposiforme, que algunos autores consideran espectros de una misma enfermedad, son tumores vasculares poco frecuentes que suelen aparecer en los primeros meses de vida y que se asocian con complicaciones hematológicas: fenómeno de Kasabach-Merritt, que puede poner en riesgo la vida del paciente.

La clasificación adecuada de los tumores vasculares es indispensable para la correcta comunicación entre las especialidades implicadas, con el fin de establecer el diagnóstico correcto y planificar el tratamiento.

PALABRAS CLAVE: Vasos linfáticos; Tumores vasculares; Malformaciones vasculares; Hemangioma congénito; Granuloma piógeno; Hemangioendotelioma; Hemangioendo-telioma kaposiforme; Fenómeno de Kasabach-Merritt.

\section{Abstract}

Vascular anomalies are a heterogeneous group of disorders that affect blood and lymphatic vessels. The International Society for the Study of Vascular Anomalies (ISSVA), classifies them in two groups: vascular tumors and vascular malformations. In this article (the first part of two), we will review the most common vascular tumors in childhood; their physiopathology, clinical manifestations, diagnosis and treatment. We will focus on infantile hemangioma, congenital hemangiomas, pyogenic granuloma, tufted angioma and kaposiform hemangioendothelioma.

Infantile hemangioma is the most frequent vascular tumor, it appears within the first 4 weeks of life and grows rapidly, to involute after the first year. It is an erythematousviolaceous, vascular neoplasm, of variable size. Congenital hemangiomas are rare, and unlike infantile hemangioma, they are fully developed at birth since their growth is intrauterine. Pyogenic granuloma is the most frequent vascular tumor, after infantile hemangioma, it is a red-violaceous, exophytic neoplasm, with a variable size; it is fast growing and bleeds easily.

Finally, tufted angioma and kaposiform hemangioendothelioma, which some authors consider as spectra of the same disease, are rare vascular tumors that usually appear in the first months of life and are associated with hematological complications such as the Kasabach-Merritt phenomenon, which may put the patient's life at risk.

Adequate classification is important for communication among the specialties involved in the care of patients in order to provide a correct diagnosis, as well as for planning treatment and understanding prognosis of the patient.

KEYWORDS: Lymphatic vessels; Vascular tumors; Vascular malformations; Congenital hemangioma; Pyogenic granuloma; Hemangioendothelioma; Kaposiform hemangioendothelioma; Kasabach-Merritt phenomenon.
${ }^{1}$ Consulta privada. Dermatóloga pediatra

${ }^{2}$ Adscrita al servicio de Dermatología.

Instituto Nacional de Pediatría, Ciudad de México.

Recibido: 6 de agosto 2019

Aceptado: 07 de noviembre 2019

Correspondencia

Carola Durán McKinster

caroladmc53@gmail.com

Este artículo debe citarse como

Morán-Villaseñor E, Campos-Cabrera BL, García-Romero MT, Durán-McKinster C. Anomalías vasculares más frecuentes en pacientes pediátricos. Parte 1 : Tumores vasculares. Acta Pediatr Mex. 2020;41(1):26-39. 


\section{INTRODUCCIÓN}

Las anomalías vasculares comprenden un amplio grupo de alteraciones en los vasos sanguíneos y linfáticos, incluidos los tumores y las malformaciones vasculares. El comportamiento y la presentación de estas lesiones son heterogéneos porque pueden aparecer en cualquier parte del cuerpo, ser congénitas o manifestarse durante los primeros años de vida. ${ }^{1,2}$

En 1982, Mulliken y Glowacki dividieron, por primera vez, las anomalías vasculares en: hemangiomas y malformaciones. ${ }^{3}$ Posteriormente, en 1996, se formó la International Society for the Study of Vascular Anomalies (ISSVA). Esta Sociedad modificó la clasificación y la dividió en tumores y malformaciones vasculares y describió la etiología y manifestaciones clínicas con base en estudios histopatológicos y de imagen. En el Cuadro 1 se muestra la clasificación de la ISSVA, actualizada en 2018. La versión completa de esta clasificación puede consultarse en www. issva.org. ${ }^{1,4}$

Los tumores vasculares se caracterizan por presentar una proliferación y la hiperplasia de células endoteliales. En contraste, las malformaciones vasculares son secundarias a errores congénitos en la morfogénesis vascular que provoca vasos malformados, ectásicos y dilatados. ${ }^{1}$

A pesar de que esta clasificación tiene más de 20 años de vigencia, y de los diversos intentos enfocados a divulgarla, hasta la fecha se siguen utilizando, erróneamente, los términos "hemangioma", "hemolinfangioma" o "linfangioma" para describir tumores vasculares y malformaciones, aludiendo solo al componente vascular, sin diferenciar entre el carácter proliferativo o malformativo propio de cada una; esto deriva en una atención inadecuada del paciente. ${ }^{5}$ De aquí la importancia de que el médico de primer contacto, el pediatra y los especialistas de las
Cuadro 1. Clasificación de las anomalías vasculares ${ }^{4}$

\begin{tabular}{|c|c|}
\hline Tumores & Malformaciones \\
\hline Benignos & Simples \\
\hline Hemangioma infantil & Capilares \\
\hline Hemangioma congénito & Linfáticas \\
\hline $\begin{array}{l}\text { Rápidamente } \\
\text { involutivo }\end{array}$ & Venosas \\
\hline $\begin{array}{l}\text { Parcialmente } \\
\text { involutivo }\end{array}$ & Arteriovenosas \\
\hline No involutivo & Fístula arteriovenosa \\
\hline $\begin{array}{l}\text { Hemangioma capilar } \\
\text { lobulado (granuloma piógeno) }\end{array}$ & Combinadas \\
\hline Angioma en penacho* & $\begin{array}{l}\text { Dos o más malforma- } \\
\text { ciones vasculares }\end{array}$ \\
\hline $\begin{array}{l}\text { Localmente agresivos o } \\
\text { limítrofes }\end{array}$ & De grandes vasos \\
\hline $\begin{array}{l}\text { Hemangioendotelioma } \\
\text { kaposiforme* }\end{array}$ & $\begin{array}{l}\text { Asociadas con otras } \\
\text { anomalías }\end{array}$ \\
\hline Sarcoma de Kaposi & $\begin{array}{l}\text { Síndrome de } \\
\text { Klippel-Trenaunay }\end{array}$ \\
\hline $\begin{array}{l}\text { Angioendotelioma papilar } \\
\text { intralinfático (tumor de Dabska) }\end{array}$ & $\begin{array}{l}\text { Síndrome de } \\
\text { Parkes-Weber }\end{array}$ \\
\hline Malignos & $\begin{array}{l}\text { Síndrome de } \\
\text { Servelle-Martorell }\end{array}$ \\
\hline Angiosarcoma & $\begin{array}{l}\text { Síndrome de } \\
\text { Sturge-Weber }\end{array}$ \\
\hline \multirow[t]{8}{*}{$\begin{array}{l}\text { Hemangioendotelioma } \\
\text { epitelioide }\end{array}$} & $\begin{array}{l}\text { Malformación capilar } \\
\text { en extremidad asociada } \\
\text { a hipertrofia congénita } \\
\text { no progresiva }\end{array}$ \\
\hline & Síndrome de Maffucci \\
\hline & $\begin{array}{l}\text { Malformación } \\
\text { capilar-macrocefalia }\end{array}$ \\
\hline & $\begin{array}{l}\text { Malformación } \\
\text { capilar-microcefalia }\end{array}$ \\
\hline & Síndrome CLOVES \\
\hline & Síndrome de Proteus \\
\hline & $\begin{array}{l}\text { Síndrome de } \\
\text { Bannayan-Rile } \\
\text { Ruvalcaba }\end{array}$ \\
\hline & Síndrome CLAPO \\
\hline
\end{tabular}

Adaptado de ISSVA Classification for Vascular Anomalies, actualizada en el 2018 y disponible en issva.org/classification. *Algunos consideran a estas 2 entidades parte de un mismo espectro, más que dos entidades distintas. 
distintas ramas de la Pediatría sean capaces de diferenciar y clasificar correctamente la afección de estos pacientes, lo que evitará tratamientos inadecuados.

En este artículo, primera parte de dos, se revisan brevemente los tumores vasculares más frecuentes en pacientes pediátricos. El enfoque se dirige a los tumores vasculares benignos porque los malignos son raros en niños.

\section{TUMORES VASCULARES BENIGNOS}

\section{Hemangioma infantil}

(Antes llamado hemangioma en fresa)

\section{Epidemiología}

Es el tumor benigno más frecuente en lactantes, afecta a $4.5 \%$ de la población general. ${ }^{6}$ Es más común en caucásicos y en el género femenino con una relación 1.4-3:1.7 Su incidencia en la población general se ha incrementado en las últimas tres décadas, en estrecha relación con la disminución tanto en la edad gestacional como en el peso al nacer. ${ }^{8}$ Está reportado que 22 a 30\% de los recién nacidos con menos de $1 \mathrm{~kg}$ de peso tienen un hemangioma. ${ }^{7}$ Otros factores de riesgo son: edad materna avanzada, embarazo múltiple, antecedente de biopsia de vellosidades coriónicas y complicaciones del embarazo: preeclampsia, placenta previa y otras anomalías placentarias. ${ }^{7}$

\section{Etiología}

Se relaciona con hipoxia local, que ocasiona sobreexpresión de factores angiogénicos: factor de crecimiento endotelial vascular (VEGF), factor de crecimiento similar a la insulina tipo 2 (IGF-2) y el transportador de glucosa 1 (GLUT-1), los 3 inducidos vía el factor inducible de hipoxia $1 \alpha$ (HIF1 $\alpha$ ). La sobreexpresión de factores angiogénicos deriva en incremento de la proliferación de células endoteliales. ${ }^{6}$ Otras hipótesis sugieren que el embolismo de angioblastos de origen placentario y la estimulación de la vía renina-angiotensina inducida por la hipoxia, contribuyen a la proliferación de células endoteliales. ${ }^{9}$

\section{Manifestaciones clínicas}

El hemangioma infantil es una neoformación vascular, casi siempre única, de coloración eritematosa o eritemato-violácea, debidamente delimitada o difusa, de tamaño variable (0.5 -30 $\mathrm{cm}$ de diámetro) (Figura 1).?

Las lesiones no suelen estar presentes al nacimiento. En algunos casos se observan lesiones precursoras constituidas por una mancha eritematosa central, rodeada de un halo pálido (Figura 2). ${ }^{6}$ El hemangioma infantil aparece en las primeras 4 semanas de vida y crece rápidamente hasta alcanzar $80 \%$ de su tamaño a los 3-5 meses; posteriormente tiene una fase de crecimiento más lenta hasta los 6-9 meses y, a partir de ese

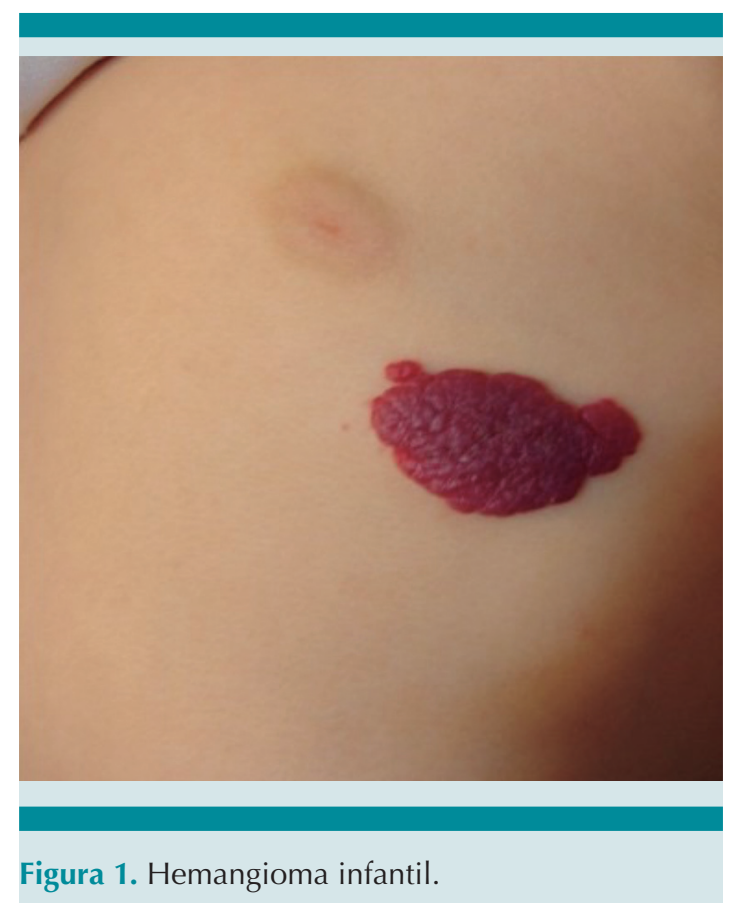




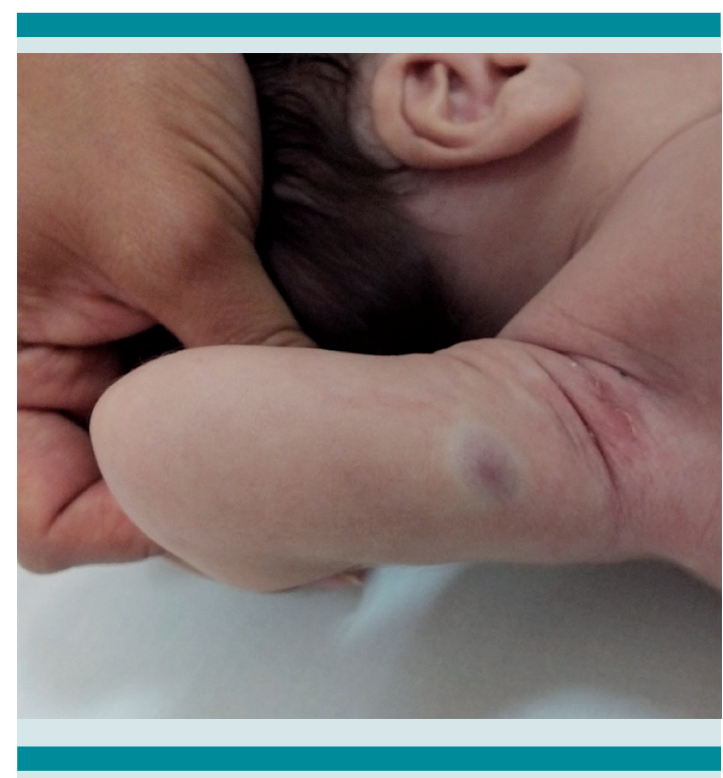

Figura 2. Lesión precursora de hemangioma infantil constituida por una mancha eritematosa central rodeada de un halo pálido.

momento, inicia una fase de involución espontánea. En 90\% de los casos hay una involución completa para los 4 años. ${ }^{6,7} \mathrm{Al}$ involucionar, 50$70 \%$ dejan cambios residuales: telangiectasias, tejido fibroadiposo, piel redundante o cambios en la pigmentación. ${ }^{6,7}$

De acuerdo con su profundidad pueden distinguirse tres subtipos: superficial (57\%), profundo (23\%) y mixto (20\%) (Figura 3 A-C). ${ }^{8}$ De acuerdo con su distribución se clasifican en: focal, segmentario, indeterminado y multifocal. ${ }^{10}$

El hemangioma infantil focal es la forma más frecuente de presentación y se caracteriza por ser una lesión única, debidamente delimitada (Figura 1). En contraste, el hemangioma infantil segmentario es más extenso, difuso y mal definido, y puede formar parte de síndromes: PHACES, LUMBAR y PELVIS. El síndrome PHACES (Figura 4) se caracteriza por la asociación de malfor-

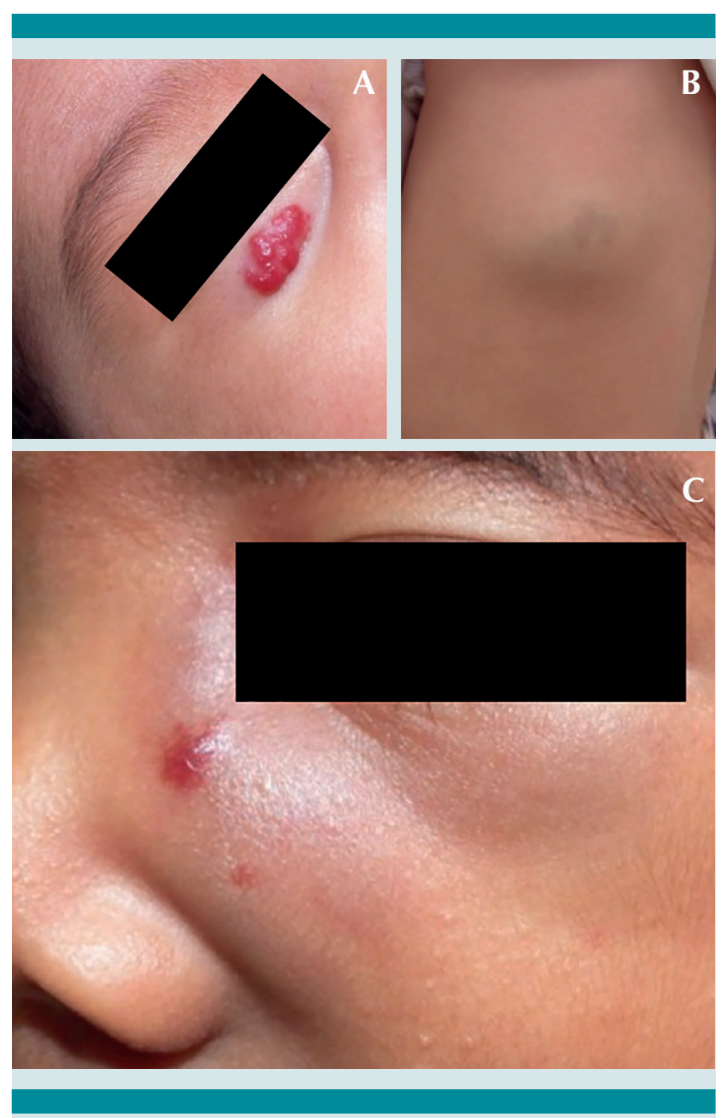

Figura 3. Hemangiomas infantiles según su profundidad. A) superficial, B) profundo, C) mixto (con componente superficial y profundo).

maciones en la fosa posterior cerebral, grandes hemangiomas faciales, anomalías anatómicas de las arterias cerebrales, coartación aórtica y otras anomalías cardiacas y oculares.

El síndrome LUMBAR se manifiesta con: hemangioma infantil en las extremidades inferiores $y$ se asocia con anormalidades urogenitales, mielomeningocele, anomalías óseas, anorrectales y renales. El síndrome PELVIS asocia hemangioma infantil perianal con malformaciones de genitales, lipomielomeningocele, anomalías vesicorrenales, ano imperforado y apéndices cutáneos (Cuadro 2). . $^{71,12}$ 
Cuadro 2. Síndromes asociados a hemangiomas infantiles segmentarios ${ }^{7}$

\begin{tabular}{|c|c|c|}
\hline PHACES & LUMBAR & PELVIS \\
\hline $\begin{array}{l}\text { Anomalías de fosa Posterior } \\
\text { Hemangioma infantil segmentario en la cara. } \\
\text { Anomalías Arteriales } \\
\text { Anomalías Cardiovasculares } \\
\text { Anomalías oculares (Eye) } \\
\text { Anomalías eSternales }\end{array}$ & $\begin{array}{l}\text { Hemangioma en la porción inferior } \\
\text { del cuerpo } \\
\text { (Lower body hemangioma) } \\
\text { Anomalías Urogenitales } \\
\text { Mielopatía (Mielomeningocele) } \\
\text { Anomalías óseas } \\
\text { (Bone deformities) } \\
\text { Malformaciones Anorrectales } \\
\text { Anomalías Renales }\end{array}$ & $\begin{array}{l}\text { Hemangioma infantil Perianal } \\
\text { Malformaciones de genitales } \\
\text { Externos } \\
\text { Lipomielo-meningocele } \\
\text { Anomalías Vesico-rrenales } \\
\text { Ano Imperforado } \\
\text { Apéndice cutáneo } \\
\text { (Skin tag) }\end{array}$ \\
\hline
\end{tabular}

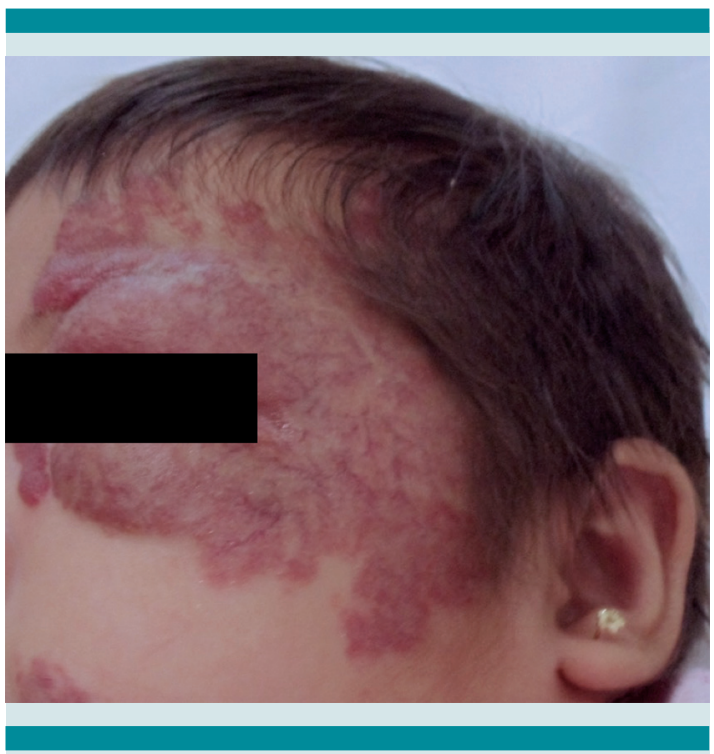

Figura 4. Hemangioma infantil segmentario en la cara, asociado con alteraciones de la fosa posterior y anomalías cardiacas, en un paciente con síndrome de PHACES.

La hemangiomatosis múltiple (o hemangioma infantil multifocal) se refiere a la coexistencia de más de 2 hemangiomas infantiles (Figura 5). Cuando el paciente tiene más de 5 hemangiomas infantiles se asocia con incidencia de $16 \%$ de hemangiomas hepáticos; por esto es indispensable el ultrasonido hepático. ${ }^{13}$

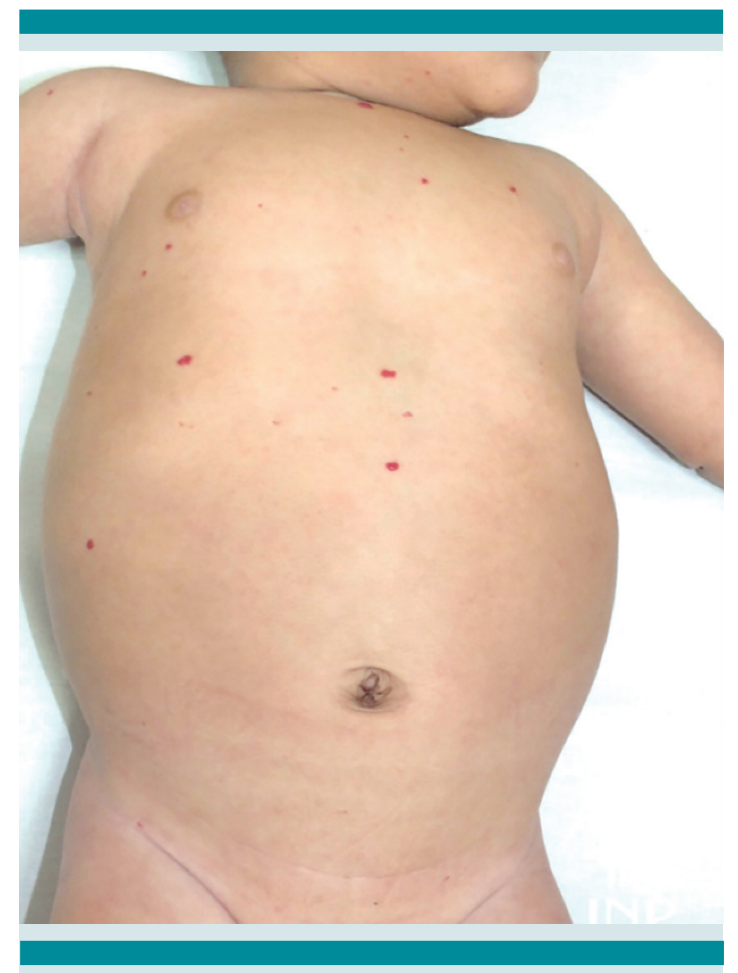

Figura 5. Hemangiomatosis múltiple.

\section{Complicaciones}

Las complicaciones se observan en alrededor de $25 \%$ de los pacientes con hemangioma infantil. Son más frecuentes en los hemangiomas infantiles grandes, segmentarios y periorificiales. El hemangioma infantil puede originar: ulceración, 


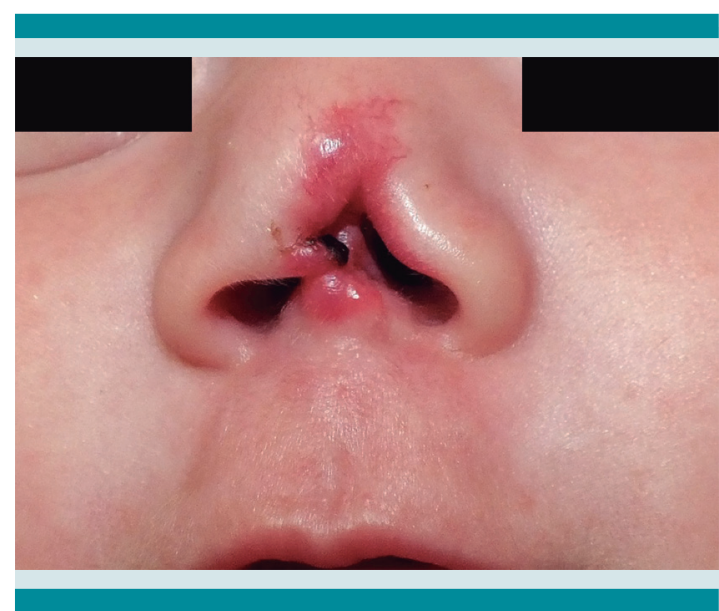

Figura 6. Hemangioma infantil en la punta nasal, con ulceración y desfiguración local.

dolor, sangrado o sobreinfección. ${ }^{7}$ Los hemangiomas infantiles perioculares pueden ocasionar ptosis palpebral y obstrucción del eje visual, lo que produce astigmatismo, anisometropía y ambliopía. Otras localizaciones periorificiales pueden dejar desfiguración local como secuela (Figura 6). ${ }^{14}$

\section{Diagnóstico}

Si bien el diagnóstico es clínico, en algunos casos, como en los hemangiomas infantiles profundos, el ultrasonido Doppler establece el diagnóstico diferencial con malformaciones vasculares. La resonancia magnética delimita la extensión en los casos que así se requiera. ${ }^{10}$

En los estudios histopatológicos se observa una proliferación no encapsulada de capilares con células endoteliales prominentes que, mediante inmunohistoquímica, puede corroborarse la positividad de la prueba para GLUT-1, CD31 y CD34. ${ }^{7,15}$

\section{Diagnóstico diferencial}

El diagnóstico diferencial de un hemangioma infantil debe establecerse con las malformaciones vasculares, sobre todo con las capilares (mancha en vino de Oporto); sin embargo, éstas son de color que va de rosa al violáceo y permanecen sin cambios. En el caso de los hemangiomas infantiles profundos la historia clásica de crecimiento gradual en el primer año de vida permite diferenciarlos de las malformaciones vasculares linfáticas. ${ }^{16}$

\section{Tratamiento}

El tratamiento depende de la localización y tipo de hemangioma infantil. Solo $1 / 3$ de los pacientes requerirá tratamiento porque los hemangiomas infantiles superficiales y pequeños en el tronco o en las extremidades, no complicados, no lo ameritan. Las indicaciones de tratamiento de pacientes con hemangioma infantil se muestran en el Cuadro 3. ${ }^{14}$

A partir del año 2008, el tratamiento de elección de los hemangiomas infantiles es el propranolol, a dosis de 2-3 mg/kg/día; ${ }^{14}$ se inicia con dosis de $1 \mathrm{mg} / \mathrm{kg} /$ día con incremento semanal hasta

Cuadro 3. Indicaciones de tratamiento en pacientes con hemangiomas infantiles ${ }^{14}$

\section{Ulcerados.}

Periorificiales (perioculares, preauriculares, en punta nasal), en los labios, el pezón o los genitales.

Que pongan en riesgo la vida (por obstrucción de la vía aérea o hemangiomas infantiles múltiples asociados con hemangiomas hepáticos).

Que ocasionen alteraciones funcionales o con riesgo de desfigurar. 
llegar a $3 \mathrm{mg} / \mathrm{kg} / \mathrm{día}$. La dosis se mantiene hasta la desaparición del hemangioma. Los resultados son excelentes o muy buenos en la mayoría de los casos y los efectos adversos escasos. ${ }^{7,17,18} \mathrm{En}$ el caso de hemangiomas infantiles pequeños, superficiales y sin complicaciones, el timolol tópico, 1-2 gotas cada 12 horas, es un tratamiento eficaz. ${ }^{10,14,19}$

\section{Hemangioma congénito}

\section{Epidemiología}

Es un tumor vascular muy raro, de incidencia desconocida. A diferencia del hemangioma infantil, no tiene predominio de género, no existe una relación con las semanas de gestación ni el peso al nacer. ${ }^{20}$

\section{Etiología}

Los hemangiomas congénitos son secundarios a mutaciones somáticas activadoras en GNAQ y GNA11 que afectan a las subunidades $\alpha$ de la proteína $\mathrm{G}$, condicionando una ganancia de función que incrementa la señalización a través de la vía de la MAP cinasa. ${ }^{21,22}$

\section{Manifestaciones clínicas}

El hemangioma congénito clínicamente se manifiesta como una neoformación vascular, casi siempre única ${ }^{22}$ y evidente desde el nacimiento. Está debidamente delimitada, con un centro que puede estar deprimido (en ocasiones incluso con ulceración central) y con un halo blanquecino en la periferia y telangiectasias en la superficie (Figura 7 A y B). ${ }^{1,20}$ Su tamaño varía de 1 a $20 \mathrm{~cm}$

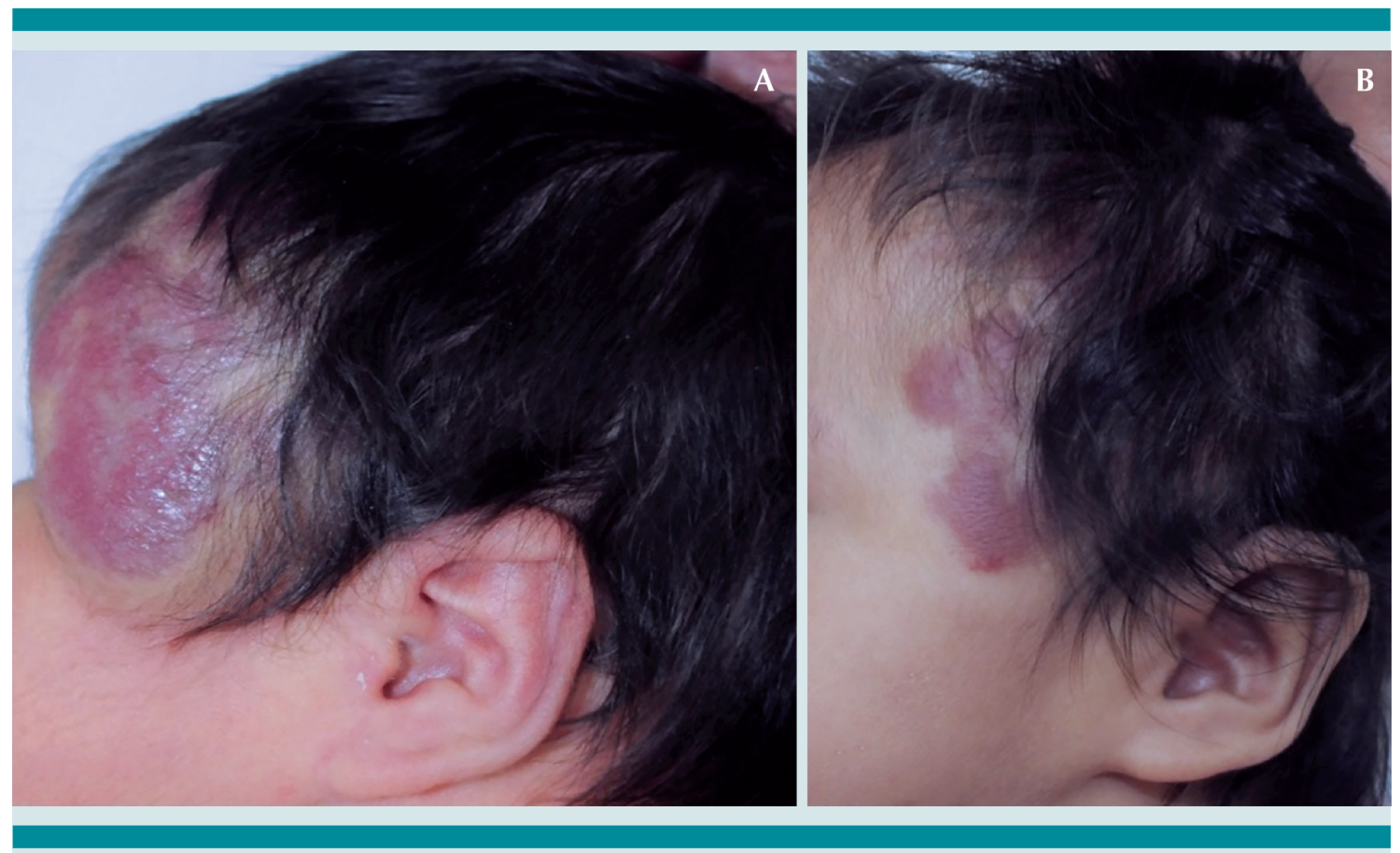

Figura 7. Hemangioma congénito rápidamente involutivo (RICH). A) $\mathrm{Al}$ nacimiento, $\mathrm{B}) \mathrm{Al}$ año de edad. 
de diámetro y es más frecuente en la cabeza, el tronco y las extremidades inferiores. ${ }^{23,24}$

A diferencia del hemangioma infantil, está completamente desarrollado al nacer, dado que su crecimiento se da en la vida intrauterina. ${ }^{23}$

Se han descrito tres tipos de hemangiomas congénitos: 1) los que permanecen sin cambios (no involutivos); 2) los que involucionan en los primeros 6-14 meses de vida (rápidamente involutivos); 3) los que involucionan parcialmente para luego mantenerse sin cambios (parcialmente involutivos)..$^{20,24}$

En la Figura 8 se muestra el comportamiento de los hemangiomas infantiles y congénitos.

\section{Complicaciones}

Los hemangiomas congénitos rápidamente involutivos pueden tener alteraciones hematológicas: trombocitopenia e, incluso, elevación de dímero-D y disminución del fibrinógeno.
Estas alteraciones son raras, leves y transitorias. $^{22,25,26}$

\section{Diagnóstico}

El diagnóstico es clínico. En el ultrasonido doppler se observa un flujo incrementado $y$ pueden identificarse incluso desde los ultrasonidos prenatales. ${ }^{20}$

La histopatología se caracteriza por una proliferación lobular de células endoteliales rodeadas de dermis fibrosa, con mayor cantidad de vasos dilatados (ectásicos) o malformados. A diferencia de los hemangiomas infantiles, estos tumores son GLUT-1 negativos. ${ }^{15,23}$

\section{Tratamiento}

Los hemangiomas congénitos rápidamente involutivos no requieren tratamiento porque desaparecen completamente en los primeros meses de vida de forma espontánea. En los casos no involutivos, o parcialmente involutivos,

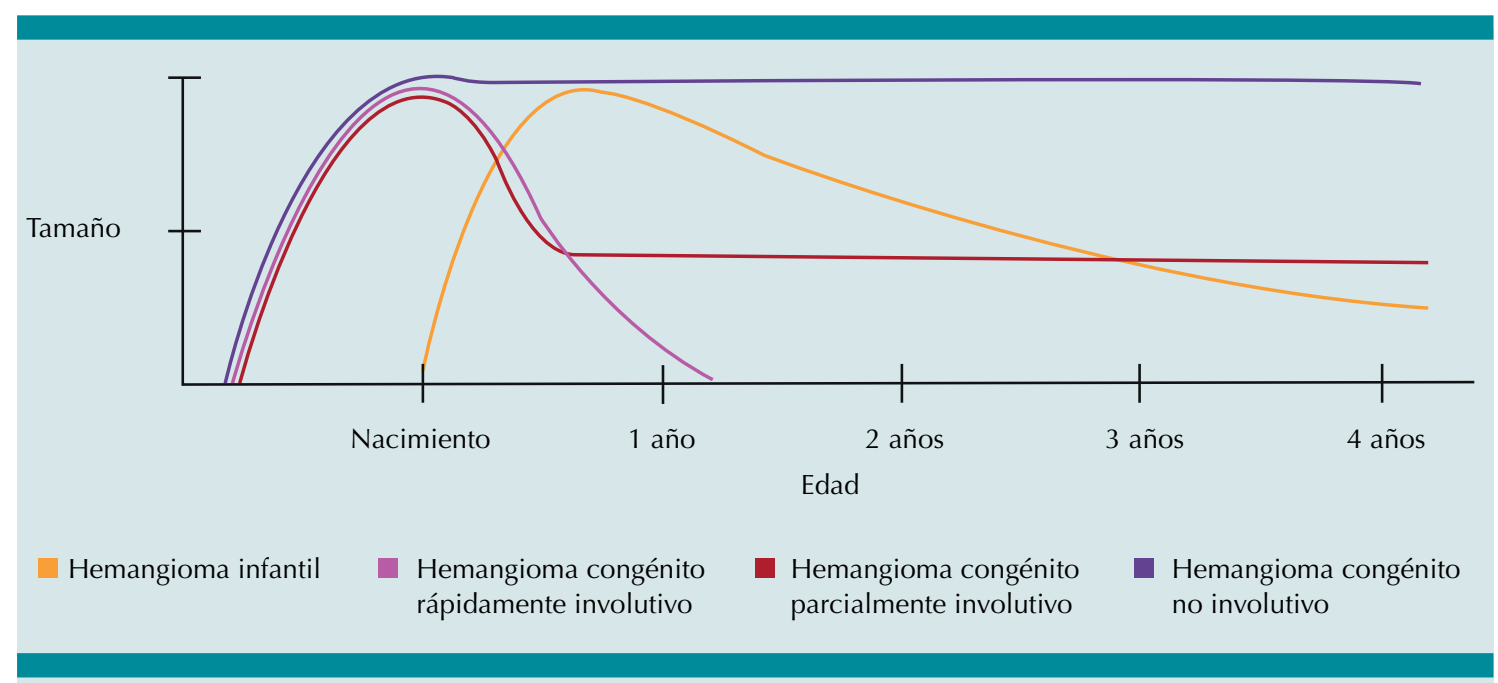

Figura 8. Evolución natural de los diferentes tipos de tumores vasculares. El hemangioma infantil crece durante el primer año de vida, para posteriormente estabilizarse e involucionar en la etapa preescolar y escolar. Los hemangiomas congénitos, por el contrario, están completamente desarrollados al nacimiento. 
está indicada la resección quirúrgica, previa embolización. El láser de colorante pulsado puede disminuir la cantidad de telangiectasias residuales. ${ }^{23,24}$

\section{Hemangioma capilar lobulado} (granuloma piógeno)

Es un tumor vascular benigno y frecuente, que afecta la piel y las mucosas. ${ }^{27}$

\section{Epidemiología}

En niños se ha reportado un ligero predominio en el género masculino, con una relación 1.5:1.1,28 La edad promedio al diagnóstico en niños es de 6 años, con límites de 4 meses a 18 años. ${ }^{28}$

\section{Etiología}

En ocasiones aparece en respuesta a traumatismos, factores hormonales y tratamientos con: antirretrovirales, retinoides tópicos y sistémicos y 5 -fluoracilo; en más de $75 \%$ de los casos no se identifican dichos factores predisponentes. ${ }^{28}$ Recientemente se describieron mutaciones somáticas activadoras en BRAF y en HRAS en estos pacientes, sobre todo en granulomas piógenos que aparecen en manchas en vino de Oporto..$^{29,30}$

\section{Manifestaciones clínicas}

Clínicamente es una neoformación de color rojovioláceo, exofítica o pediculada, debidamente delimitada, que varía de 1 a $10 \mathrm{~mm}$ de diámetro (Figura 9). Son de crecimiento rápido y sangran fácilmente, datos que facilitan el diagnóstico diferencial con otras lesiones. ${ }^{28}$ Predominan en la cabeza y el cuello (más del $70 \%$ de los casos), aunque pueden encontrarse en cualquier parte del cuerpo. ${ }^{28}$

Lo común es que sean lesiones únicas; ${ }^{28}$ sin embargo, se han reportado lesiones múltiples, sobre todo en pacientes con quemaduras extensas. ${ }^{31}$

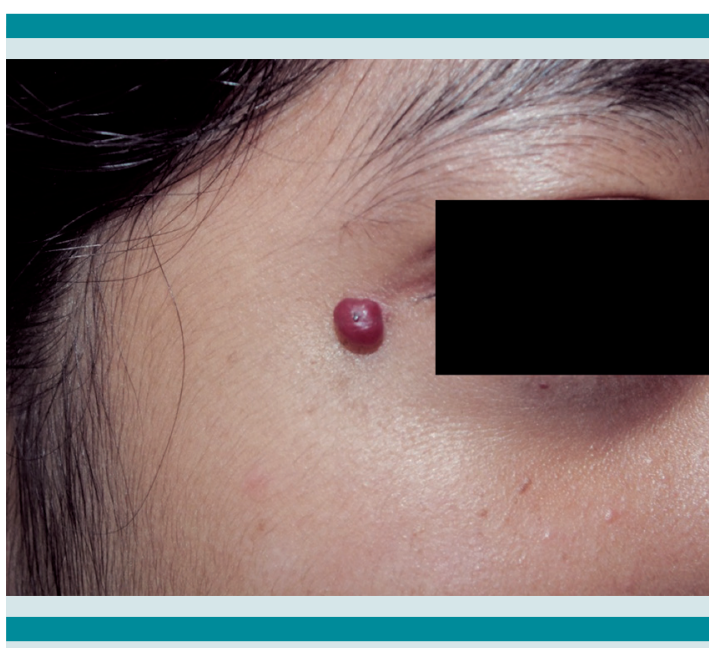

Figura 9. Hemangioma capilar lobulado.

\section{Diagnóstico}

El diagnóstico es clínico. En la histopatología se identifica una neoformación de aspecto polipoide constituida por una proliferación lobular de capilares dilatados, separados por septos de tejido fibroso. La epidermis suprayacente está adelgazada e, incluso, ulcerada y con un coIlarete epitelial que delimita la neoformación. Al igual que los hemangiomas congénitos, los capilares son GLUT-1 negativos. ${ }^{15}$

\section{Diagnóstico diferencial}

En niños, el principal diagnóstico diferencial es con el hemangioma infantil; sin embargo, la evolución permite diferenciarlos fácilmente. En adolescentes y adultos el diagnóstico diferencial incluye: melanoma amelánico, poroma écrino y carcinoma basocelular, por lo que en este grupo etario el estudio histopatológico es decisivo. ${ }^{28}$

\section{Tratamiento}

La resección y cauterización son el tratamiento de elección, aunque puede haber recidivas en 
caso de resección incompleta. ${ }^{28}$ También están indicados los beta-bloqueadores: timolol y propranolol, con menor porcentaje de eficacia. . $2,33,34^{2}$

\section{Angioma en penacho (tufted angioma)}

El angioma en penacho, también conocido como angioblastoma de Nakagawa, es un tumor vascular benigno, poco frecuente. ${ }^{35}$

\section{Epidemiología}

La incidencia exacta de esta neoplasia se desconoce. En 15\% de los pacientes la lesión está presente al nacimiento, en $50 \%$ aparece antes del año de vida y en $80 \%$ en los primeros 10 años. ${ }^{35,36}$

\section{Etiología}

Su etiopatogenia se relaciona con incremento de la secreción local de factores de crecimiento involucrados en la angiogénesis, como IL-8. Puesto que las células fusiformes que componen el tumor expresan marcadores linfáticos (D2-40 y PROX1), se considera que deriva de vasos linfáticos. ${ }^{15,35}$

\section{Manifestaciones clínicas}

La presentación clínica es muy variable: ${ }^{36}$ una placa o un nódulo infiltrado, eritematovioláceo, o en algunas ocasiones color marrón, de consistencia firme, mal delimitado, de aproximadamente $1-10 \mathrm{~cm}$ de diámetro. ${ }^{1,35}$ Las lesiones suelen ser únicas y predominan en las extremidades y el tronco (Figura 10 A y B).

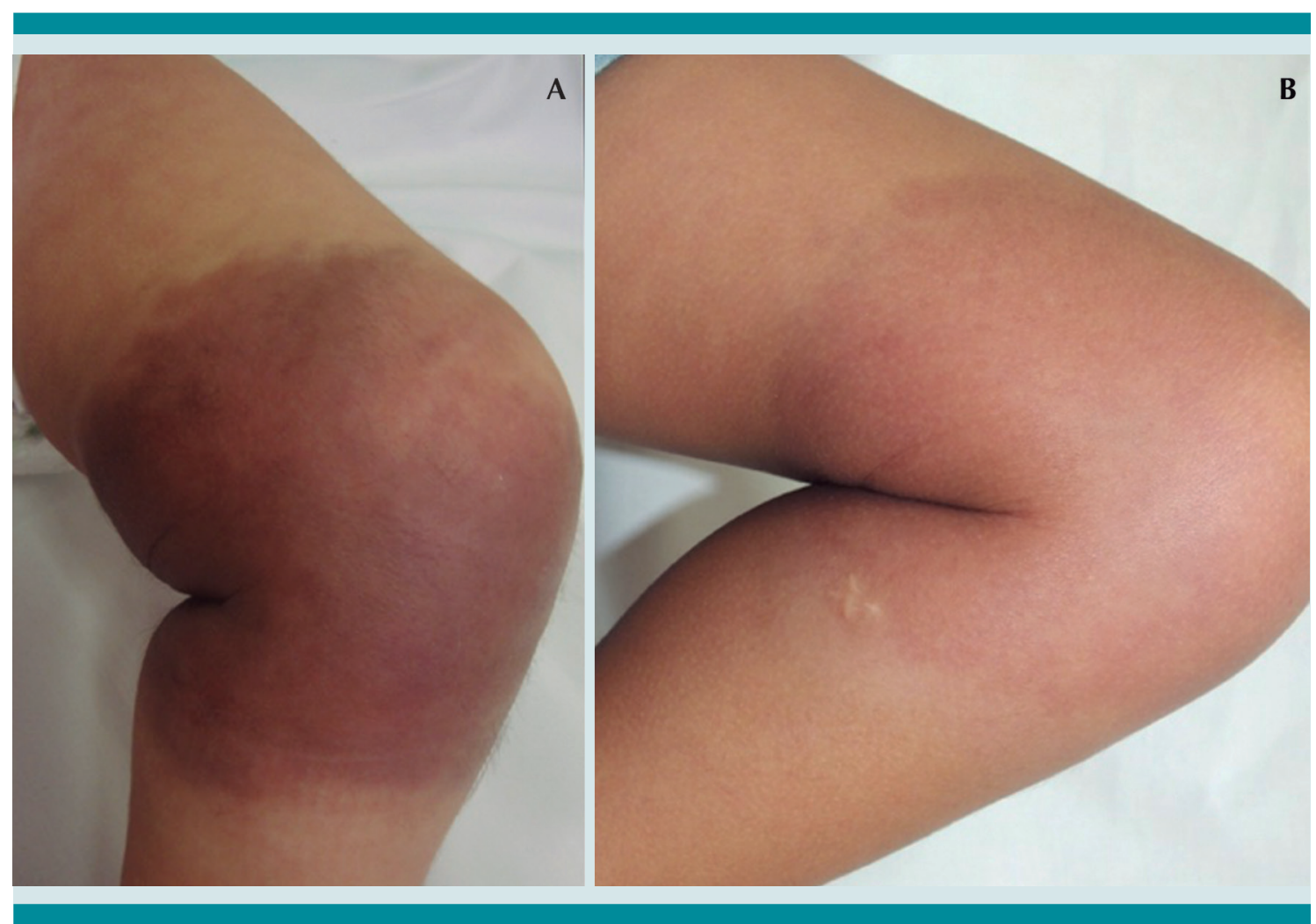

Figura 10. Angioma en penacho. A) Inicial, B) Final. 
Característicamente la piel suprayacente tiene hiperhidrosis e hipertricosis. ${ }^{1,35,36}$

Su evolución también es variable, en la mayoría de los casos hay regresión espontánea antes de los dos años del inicio; sin embargo, otros pueden mostrar poca tendencia a la regresión. ${ }^{37}$

\section{Complicaciones}

El 10\% de los pacientes con angioma en penacho tiene fenómeno de Kasabach-Merritt, es decir, una coagulopatía por consumo, con trombocitopenia severa, disminución de fibrinógeno e incremento de dímero $\mathrm{D}$, con o sin anemia microangiopática. ${ }^{38}$

\section{Diagnóstico}

La biopsia de piel muestra numerosos capilares que forman nódulos, lo que da un aspecto de "balas de cañón". En la dermis superficial y profunda están rodeados de espacios vasculares vacíos, semilunares, con forma de hendidura. Estos capilares son GLUT-1 negativos, positivos para podoplanina (D2-40), PROX1, CD31 y CD34.,115

\section{Diagnóstico diferencial}

El principal diagnóstico diferencial es con el hemangioendotelioma kaposiforme. Algunos autores consideran a estos dos tumores parte de un mismo espectro. El angioma en penacho es la forma más leve y el hemangioendotelioma kaposiforme la más severa. El dato que puede orientar hacia un hemangioendotelioma kaposiforme es la lesión que aparece en el primer mes de vida, de crecimiento rápido, de coloración violácea, que afecta los tejidos más profundos y que se asocia con mayor frecuencia con fenómeno de Kasabach-Merritt (70 vs 10\% en angioma en penacho). ${ }^{1,35}$

\section{Tratamiento}

Los casos que requieren tratamiento sistémico son los que cursan con fenómeno de KasabachMerritt, limitación funcional o afectación de órganos vitales. Los esteroides sistémicos son el tratamiento de primera línea, a dosis de 2-3 mg/ $\mathrm{kg} /$ día. En casos resistentes o con coagulopatía importante, la vincristina ha demostrado ser muy eficaz. Sirolimus (inhibidor de la vía mTOR) ha demostrado eficacia, con mínimos efectos colaterales. ${ }^{39}$

\section{TUMORES VASCULARES LOCALMENTE AGRESIVOS}

\section{Hemangioendotelioma kaposiforme}

Epidemiología

Es un tumor vascular raro, que aparece en 0.9 de cada 100,000 recién nacidos vivos y afecta por igual a hombres y mujeres. En $60 \%$ de los casos, la lesión se inicia en el primer mes de vida y puede afectar la piel, el retroperitoneo y otros órganos. ${ }^{2,40}$

\section{Etiología}

Comparte etiopatogenia con el angioma en penacho, en ambos casos se propone que las lesiones derivan del endotelio linfático, por su positividad, mediante inmunohistoquímica, para vasos linfáticos (D2-40 y PROX1). ${ }^{15}$

\section{Manifestaciones clínicas}

Es una neoformación vascular, única, subcutánea, de coloración eritematoviolácea, de consistencia firme, indurada y mal delimitada, de crecimiento rápido, de $5 \mathrm{a} 10 \mathrm{~cm}$ hasta abarcar toda una extremidad (Figura 11 A y B). Se localiza con más frecuencia en el tronco y las extremidades. $^{2}$ 


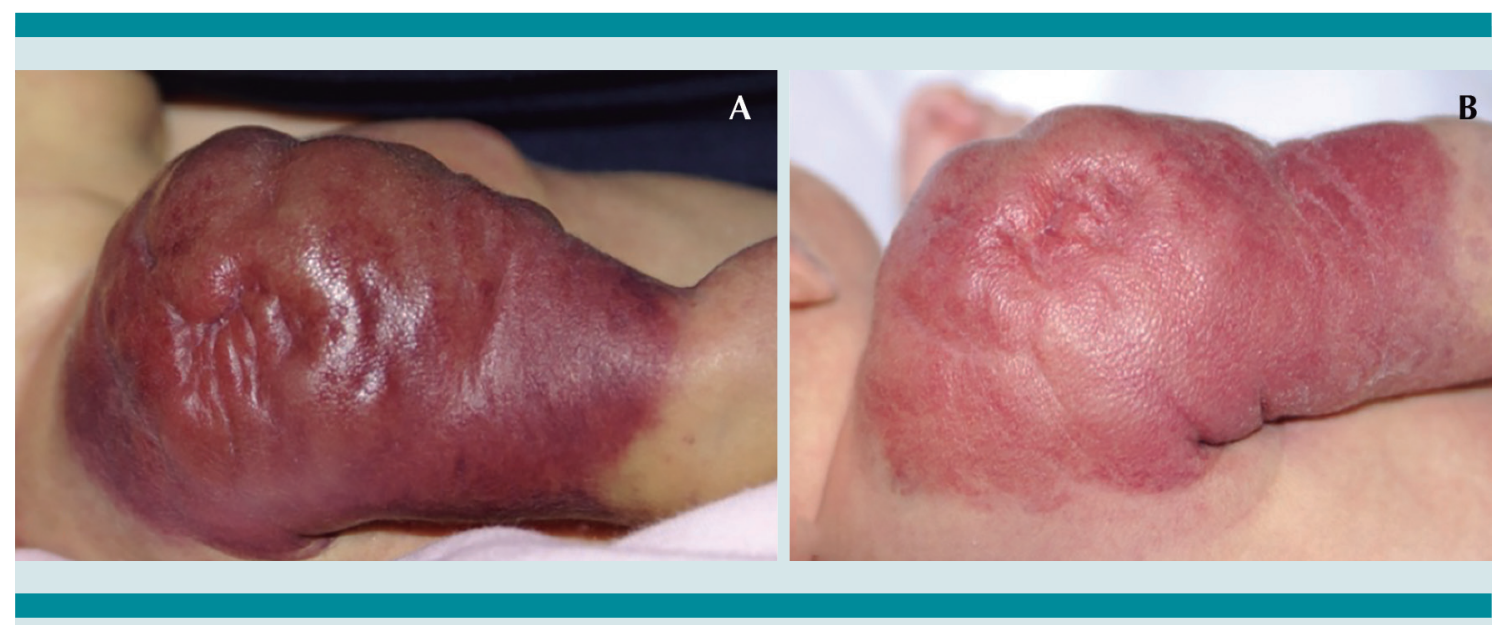

Figura 11. Hemangioendotelioma kaposiforme. A) Inicial, B) Posterior a 1 semana de tratamiento con prednisona a $3 \mathrm{mg} / \mathrm{kg} /$ día.

\section{Complicaciones}

El $70 \%$ de los pacientes con hemangioendotelioma kaposiforme tiene fenómeno de Kasabach-Merritt; sin embargo, en los casos en los que solo se localiza en el retroperitoneo o la cavidad intratorácica, sin lesiones cutáneas, el porcentaje de coagulopatía se incrementa a 85 y $100 \%$, respectivamente. ${ }^{40}$

\section{Diagnóstico}

En la histopatología se observa proliferación de células fusiformes que infiltran la dermis y el tejido celular subcutáneo, a veces también el músculo. Estas células son GLUT-1 negativo, pero D2-40 y PROX1 positivas, al igual que en el caso del angioma en penacho. ${ }^{2,15,41}$

\section{Tratamiento}

Por la elevada frecuencia de complicaciones, los pacientes con hemangioendotelioma kaposiforme deben tratarse con esteroides sistémicos, a dosis de 2-3 mg/kg/día, solos o en combinación con vincristina. Al igual que en el angioma en penacho, el sirolimus por vía oral ha demostrado eficacia, con escasos efectos adversos. ${ }^{39,42-44}$

En la actualidad, la cirugía no se considera un tratamiento útil porque los márgenes del tumor están mal delimitados y la probabilidad de recurrencia y complicaciones (sobre todo sangrado) es alta. ${ }^{2}$

En pacientes con hemangioendotelioma kaposiforme no se recomienda transfundir plaquetas, excepto cuando hay sangrado activo o previo a un procedimiento quirúrgico, porque la transfusión de plaquetas incrementa la coagulopatía e, incluso, el volumen del tumor. Se recomiendan crioprecipitados o plasma fresco congelado si el fibrinógeno es menor de $1 \mathrm{~g} / \mathrm{dL} .{ }^{2,41}$

\section{CONCLUSIONES}

Los tumores vasculares, específicamente el hemangioma infantil, constituyen un motivo frecuente de consulta al pediatra, razón para estar familiarizado con ellos. Cada tumor tiene características clínicas, radiológicas e histopatológicas propias que permiten diferenciarlos entre sí. Conocer esas características permite establecer 
el diagnóstico correcto de forma temprana y, por consiguiente, indicar un tratamiento adecuado y oportuno, y mejorar el pronóstico del paciente. Si los tumores vasculares comprenden un amplio grupo de entidades clínicas cuyas manifestaciones dependen de la topografía, tamaño y complicaciones, es conveniente tratar a esos pacientes en grupos interdisciplinarios, para establecer debidamente el diagnóstico e indicar el tratamiento correcto.

\section{REFERENCIAS}

1. Wassef M, Blei F, Adams D, Alomari A, Baselga E, Berenstein $A$, et al. Vascular anomalies classification: recommendations from the International Society for the Study of Vascular Anomalies. Pediatrics. 2015;136(1):e203-14.

2. Foley LS, Kulungowski AM. Vascular anomalies in pediatrics. Adv Pediatr. 2015;62(1):227-55.

3. Mulliken JB, Glowacki J. Hemangiomas and vascular malformations in infants and children: a classification based on endothelial characteristics. Plast Reconstr Surg. 1982;69(3):412-22.

4. International Society for the Study of Vascular Anomalies. En: http://www.issva.org

5. Pahl KS, Kim K, Sams C, Alvarez H, Smith SV, Blatt J. Inconsistency in classifying vascular anomalies: What's in a name? Pediatr Blood Cancer. 2018;65(3). doi:10.1002/pbc.26836.

6. Léauté-Labrèze $\mathrm{C}$, Harper $\mathrm{JI}$, Hoeger PH. Infantile haemangioma. Lancet. 2017;390(10089):85-94.

7. Darrow DH, Greene AK, Mancini AJ, Nopper AJ. Diagnosis and management of infantile hemangioma. Pediatrics. 2015;136(4):e1060-104.

8. Anderson KR, Schoch JJ, Lohse CM, Hand JL, Davis DM, Tollefson MM. Increasing incidence of infantile hemangiomas (IH) over the past 35 years: Correlation with decreasing gestational age at birth and birth weight. J Am Acad Dermatol. 2016;74(1):120-6.

9. Smith CJF, Friedlander SF, Guma M, Kavanaugh A, Chambers $C D$. Infantile hemangiomas: An updated review on risk factors, pathogenesis, and treatment. Birth Defects Res. 2017;109(11):809-15.

10. Püttgen KB. Diagnosis and management of infantile hemangiomas. Pediatr Clin North Am. 2014;61(2):383-402.

11. Garzon MC, Epstein LG, Heyer GL, Frommelt PC, Orbach DB, Baylis AL, et al. PHACE Syndrome: Consensusderived diagnosis and care recommendations. J Pediatr. 2016;178:24-33.e2.

12. lacobas I, Burrows PE, Frieden IJ, Liang MG, Mulliken JB, Mancini AJ, et al. LUMBAR: association between cutaneous infantile hemangiomas of the lower body and regional congenital anomalies. J Pediatr. 2010;157(5):795-801.e1-7.
13. Rialon KL, Murillo R, Fevurly RD, Kulungowski AM, Zurakowski $\mathrm{D}$, Liang $\mathrm{M}$, et al. Impact of screening for hepatic hemangiomas in patients with multiple cutaneous infantile hemangiomas. Pediatr Dermatol. 2015;32(6):808-12.

14. Krowchuk DP, Frieden IJ, Mancini AJ, Darrow DH, Blei $F$, Greene AK, et al. Clinical practice guideline for the management of infantile hemangiomas. Pediatrics 2019;143(1):pii:e20183475.

15. Johnson EF, Davis DM, Tollefson MM, Fritchie K, Gibson LE. Vascular tumors in infants: case report and review of clinical, histopathologic, and immunohistochemical characteristics of Infantile Hemangioma, Pyogenic Granuloma, Noninvoluting Congenital Hemangioma, Tufted Angioma, and Kaposiform Hemangioendothelioma. Am J Dermatopathol. 2018;40(4):231-9.

16. Garzon MC, Huang JT, Enjolras O, Frieden IJ. Vascular malformations: Part I. J Am Acad Dermatol. 2007;56(3):353-70.

17. Léauté-Labrèze $C$, Dumas de la Roque $E$, Hubiche $T$, Boralevi F, Thambo JB, Taïeb A. Propranolol for severe hemangiomas of infancy. N Engl J Med. 2008;358(24):2649-51.

18. Drolet BA, Frommelt PC, Chamlin SL, Haggstrom A, Bauman NM, Chiu YE, et al. Initiation and use of propranolol for infantile hemangioma: report of a consensus conference. Pediatrics. 2013;131(1):128-40.

19. Dalla Costa R, Prindaville B, Wiss K. Doing the math: A simple approach to topical timolol dosing for infantile hemangiomas. Pediatr Dermatol. 2018;35(2):276-7.

20. Mulliken JB, Enjolras O. Congenital hemangiomas and infantile hemangioma: missing links. J Am Acad Dermatol. 2004;50(6):875-82.

21. Ayturk UM, Couto JA, Hann S, Mulliken JB, Williams KL, Huang $A Y$, et al. Somatic activating mutations in GNAQ and GNA11 are associated with congenital hemangioma. Am J Hum Genet. 2016;98(6):1271. doi:10.1016/j. ajhg.2016.05.010.

22. Funk $T$, Lim $Y$, Kulungowski $A M$, Prok $L$, Crombleholme TM, Choate $\mathrm{K}$, et al. Symptomatic congenital hemangioma and congenital hemangiomatosis associated with a somatic activating mutation in GNA11. JAMA Dermatol. 2016;152(9):1015-20.

23. Lee PW, Frieden IJ, Streicher JL, McCalmont T, Haggstrom AN. Characteristics of noninvoluting congenital hemangioma: a retrospective review. J Am Acad Dermatol. 2014;70(5):899-903.

24. Nasseri E, Piram M, McCuaig CC, Kokta V, Dubois J, Powell J. Partially involuting congenital hemangiomas: a report of 8 cases and review of the literature. J Am Acad Dermatol. 2014;70(1):75-9.

25. Rangwala $S$, Wysong $A$, Tollefson $M M$, Khuu P, Benjamin LT, Bruckner AL. Rapidly involuting congenital hemangioma associated with profound, transient thrombocytopenia. Pediatr Dermatol. 2014;31(3):402-4.

26. Baselga E, Cordisco MR, Garzon M, Lee MT, Alomar A, Blei F. Rapidly involuting congenital haemangioma associated 
with transient thrombocytopenia and coagulopathy: a case series. Br J Dermatol. 2008;158(6):1363-70.

27. Mills SE, Cooper PH, Fechner RE. Lobular capillary hemangioma: the underlying lesion of pyogenic granuloma. A study of 73 cases from the oral and nasal mucous membranes. Am J Surg Pathol. 1980;4(5):470-9.

28. Pagliai KA, Cohen BA. Pyogenic granuloma in children. Pediatr Dermatol. 2004;21(1):10-3.

29. Lim YH, Douglas SR, Ko CJ, Antaya RJ, McNiff JM, Zhou J, et al. Somatic activating RAS mutations cause vascular tumors including pyogenic granuloma. J Invest Dermatol. 2015;135(6):1698-700.

30. Groesser L, Peterhof E, Evert M, Landthaler M, Berneburg M, Hafner C. BRAF and RAS mutations in sporadic and secondary pyogenic granuloma. J Invest Dermatol. 2016;136(2):481-6.

31. Zhao H, Zhang C, Fu X. Multiple Pyogenic Granulomas After Burns: Response to conservative treatment in five Children. Pediatr Dermatol. 2015;32(4):e175-6.

32. Knöpfel N, Escudero-Góngora MDM, Bauzà A, Martín-Santiago A. Timolol for the treatment of pyogenic granuloma (PG) in children. J Am Acad Dermatol. 2016;75(3):e105-e6.

33. Gupta $D$, Singh N, Thappa DM. Is timolol an effective treatment for pyogenic granuloma? Int J Dermatol. 2016;55(5):592-5.

34. Neri I, Baraldi C, Balestri R, Piraccini BM, Patrizi A. Topical $1 \%$ propranolol ointment with occlusion in treatment of pyogenic granulomas: An open-label study in 22 children. Pediatr Dermatol. 2018;35(1):117-20.

35. Victoria Martínez AM, Cubells Sánchez L, Esteve Martínez A, Estela Cubells JR, Febrer Bosch I, Alegre de Miquel V, et al. Tufted angiomas in childhood: A series of 9 cases and a literature review. An Pediatr (Barc). 2015;83(3):201-8.
36. Herron MD, Coffin CM, Vanderhooft SL. Tufted angiomas: variability of the clinical morphology. Pediatr Dermatol. 2002;19(5):394-401.

37. Ishikawa $\mathrm{K}$, Hatano $\mathrm{Y}$, Ichikawa $\mathrm{H}$, Hashimoto $\mathrm{H}$, Fujiwara $\mathrm{S}$. The spontaneous regression of tufted angioma: A case of regression after two recurrences and review of 27 cases reported in the literature. Dermatology 2005; 210(4):346-8.

38. Kelly M. Kassabach-Merritt phenomenon. Pediatr Clin North Am. 2010;57(5):1085-9.

39. Liu X, Li J, Qu X, Yan W, Zhang L, Zhang S, et al. Clinical Outcomes for Systemic Corticosteroids Versus Vincristine in Treating Kaposiform Hemangioendothelioma and Tufted Angioma. Medicine (Baltimore). 2016;95(20):e3431.

40. Croteau SE, Liang MG, Kozakewich HP, Alomari Al, Fishman SJ, Mulliken JB, et al. Kaposiform hemangioendothelioma: atypical features and risks of Kassabach-Merritt phenomenon in 107 referrals. J Pediatr. 2013;162(1):142-7.

41. O'Rafferty C, O'Regan GM, Irvine AD, Smith OP. Recent advances in the pathobiology and management of Kassabach-Merritt phenomenon. Br J Haematol. 2015;171(1):38-51.

42. Wang H, Duan $Y$, Gao $Y$, Guo X. Sirolimus for vincristineresistant Kassabach-Merritt phenomenon: Report of eight patients. Pediatr Dermatol. 2017;34(3):261-5.

43. Peng $\mathrm{S}$, Yang $\mathrm{K}, \mathrm{Xu} Z \mathrm{Z}$, Chen S, Ji Y. Vincristine and sirolimus in the treatment of kaposiform haemangioendothelioma. J Paediatr Child Health. 2019. doi:10.1111/jpc.14370. [Epub ahead of print]

44. Mariani LG, Schmitt IR, Garcia CD, Kiszewski AE. Low dose sirolimus treatment for refractory tufted angioma and congenital kaposiform hemangioendothelioma, both with Kassabach-Merritt phenomenon. Pediatr Blood Cancer. 2019. doi:10.1002/pbc.27810. [Epub ahead of print] 